\title{
Corn Oil Concentrations Detection for Food Industry Research Development by Using Application of Fiber Optic Liquid Sensor Concept
}

\author{
Md Ashadi Md Johari ${ }^{1, *}$, Aziean Mohd Azize ${ }^{1}$, Rahaini Mohd Said ${ }^{1}$, Nor Azazi Ngatiman ${ }^{1}$, \\ Noradila Zaine ${ }^{1}$ \\ ${ }^{1}$ Faculty of Engineering Technology, Universiti Teknikal Malaysia Melaka, Hang Tuah Jaya, 76100 \\ Durian Tunggal, Melaka, Malaysia
}

\begin{abstract}
This paper purposed application of fiber optic as liquid sensor for detection of various concentrations of corn oil. The final result would an additional understanding about fiber optic sensor and could be used in related field such as food industry sector. They are 20 samples of corn oil with different concentrations between $0 \%$ until $100 \%$ are tasted. For every concentration, would experience a dipped process together with fiber optic before measuring process. Each of concentrations would present different results in line graph. The experiment results would be discussed in listed values of sensitivity, correlation and coefficient of determination of graft which are totally dependent on concentration of corn oil and the light source. With a different value of concentration and $1550 \mathrm{~nm}$ wavelength of light source, the maximum sensitivity of 0.00072 the coefficient of determination $94.0 \%$ is obtained at third slope, respectively. Fiber optic is successfully to be corn oil liquid sensor and would be in high performance by using $1550 \mathrm{~nm}$ wavelength of light source and measuring concentration more than $85 \%$.
\end{abstract}

\section{Introduction}

Fiber optic has become one of the most influence technologies in communication engineering recently [1]. Fiber optic cable already tasted which able to convey large bundle of data transmission in one time, causing to be use in other field rather than communication [2 - 3]. Originally, fiber optic cable was known as bundle of glass which able to transmit modulated light wave as massage data. Its capability has been tasted over years which are more advantage than metal cable in data transmissions [5]. The biggest advantage of optical fiber is the fact it is the most cost effective means of transporting information. Fiber can transport more information longer distances in less time than any other communication medium. The bandwidth and distance capability of fiber means that fewer cables, fewer

* Corresponding author: ashadi@utem.edu.my 
repeaters, less power and less maintenance are needed. In addition, fiber is unaffected by the interference of electromagnetic radiation which makes it possible to transmit information and data with less noise and less error. These perhaps would be evidences for fiber as a best medium in data transmission. However, this paper would present implementation of fiber optic cable as liquid sensor. The light travelled inside fiber optic would be interfered by existing of liquid molecules around fiber cable. This would be the result of experiment because each of concentrations would consist of different molecules and different value of light source travelled.

Corn oil is oil extracted from the germ of corn. Its main use is in cooking, where its high smoke point makes refined corn oil valuable frying oil. Corn oil is also a feedstock used for biodiesel. Other industrial uses for corn oil include soap, salve, paint, rust proofing for metal surfaces, inks, textiles and insecticides. It is sometimes used as a carrier for drug molecules in pharmaceutical preparations. However, this experiment is to analyze sensitivity of fiber optic sensor in determine the concentration of corn oil. It is because only right concentration can be used in certain purpose such as in chemical industry.

There a various tremendous experiment conducted recently regarding liquid concentration measurement [4][6]. Be that as it may, without precedent for this trial, it was involve the fiber optic link as a fundamental segment in the olive oil detecting action. This examination maybe could help another strategy to detecting fluid focus for different scientists. Decent ramifications behind this trial would be to recognize the ability of fiber optic in fluid fixation detecting action.

The final result from this experiment would produce few different graphs. Each of graphs would be analyzed for correlation $R$ value, coefficient of determination $\left(R^{2}\right)$ value and also sensitivity value. Correlation $(\mathrm{R})$ value would represent a relationship between corn oil concentrations with voltage value. The value would be perfect if it is close to 1.0, which is mean that the changes in oil concentrations would affect the voltage value. Coefficient of determination $\left(\mathrm{R}^{2}\right)$ value is a percentage of corn oil will affect the final voltage value. In other words, the higher percentage value could cause larger influence of corn oil towards final voltage value. These three values would determine the best performance of fiber optic as liquid sensor towards different concentrations of coil oil.

\section{Experiment setup}

The experiment schematic showed in Fig.1.1, consist of light source, silicon detector, amplifier and work station (computer). The main component in this experiment is fiber optic cable which is separated into two parts. The fiber optic cable is made from plastic with core diameter is 0.25 and NA is 0.35 . The first part is connected from light source to container full with corn oil liquid and the second part is from container to the silicon detector. The importance area between of these 2 parts is in middle of fiber cable; which is a sensor activity will happen at this area. It is a same fiber cable; however it has been stripped using appropriate stripper to make it thinner than other part of fiber. This procedure is to make that part more sensitive to liquid particles. In other words, the more thinner of diameter, the more sensitive fiber optic would be and its capability to sense particle of corn oil liquid would be grater. Light source used would produce several different of wavelengths which are $850 \mathrm{~nm}, 1300 \mathrm{~nm}, 1310 \mathrm{~nm}$ and $1550 \mathrm{~nm}$. However due to high demand of corn oil, only $1310 \mathrm{~nm}$ and $1500 \mathrm{~nm}$ would be use as main light sources. The middle stripped fiber will be dipped inside a container full with coil oil liquid in several times and normally more than an hour. This procedure is to ensure that fiber optic cable could react to the coil oil liquid particle. However, only one concentration will be use in one time. In other word, this experiment will be repeated by using different 
concentrations. Before change to another concentration, fiber would be taken out from container and would be clean using an alcohol liquid.

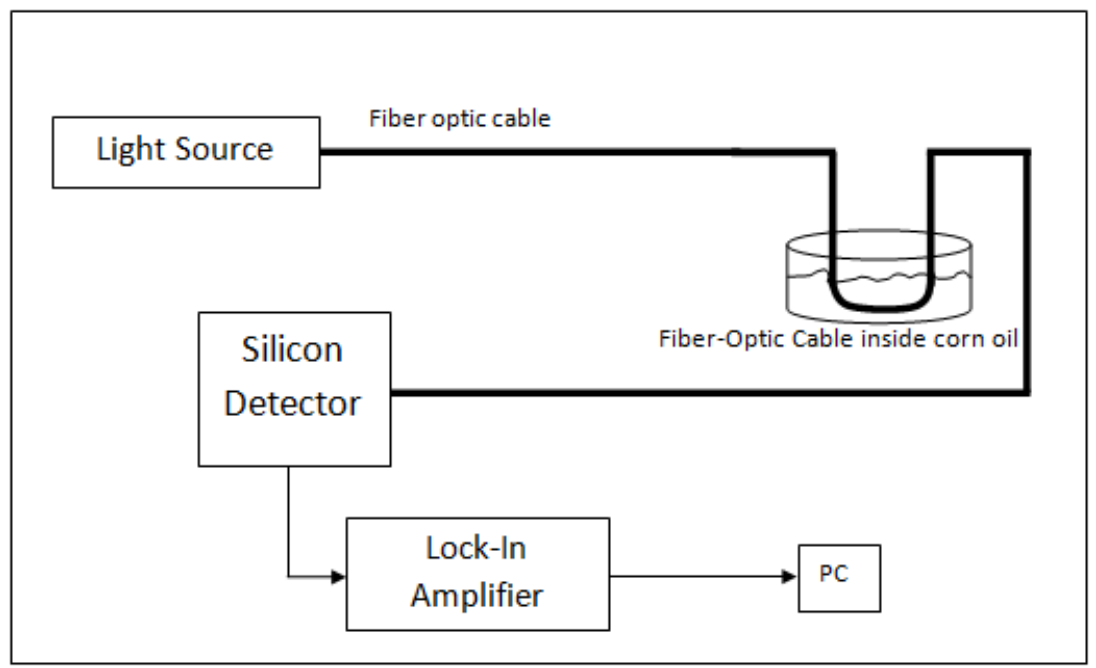

Fig. 1. Schematic diagram of the proposed fiber-optic sensor

The modulated light source would be transmitted inside fiber optic cable from beginning until silicon detector. However, during light travelling, it will experience uneven situation right after reached at stripped area which is inside corn oil liquid. This is because; the light travelling process will be disturbed by coil oil particle which are presence around stripped area. Each concentrations of corn oil will give different impact to the light source. Light travelled finally reached to silicon detector for filtering process and transfer to amplifier for amplification process. The final result would be in decibel (dB) and will be plotted into line graph in the computer.

\section{Results and discussion}

Fig.2 shows the overall result from this experiment. There are two different line in this graph representing two types of light source (different wavelength) used in this experiment. Coil oil liquid has been sampled into 20 different concentrations started from $0 \%$ of concentration until $100 \%$. The line graphs are plotted based on different concentrations. In other words, every concentration would produce different final result. The output in this experiment is in decibel; converted into output voltage. The first experiment started with $1310 \mathrm{~nm}$ of wavelength. Begun with $0 \%$ of concentration, the line graph showed down trend until it reached to $65 \%$ of concentration. However, the line graph showed up trend from that point until $100 \%$ of concentration. This experiment is continued with $1550 \mathrm{~nm}$ wavelength used as main light source. There are three different trend showed in the graph which are down trend from beginning until $40 \%$ of concentration, uptrend from $50 \%$ to $80 \%$ of concentrations and finally back to down trend from concentration $85 \%$ until $100 \%$. Based on the results, by used $1310 \mathrm{~nm}$ as light source, there would be two slope in the line graph would be sampled for further analysis. However for $1550 \mathrm{~nm}$ of light source, three different slopes would be used for analysis. This analysis will determine which of the slopes give major contribution to the performance of fiber optic as corn oil liquid sensor. 


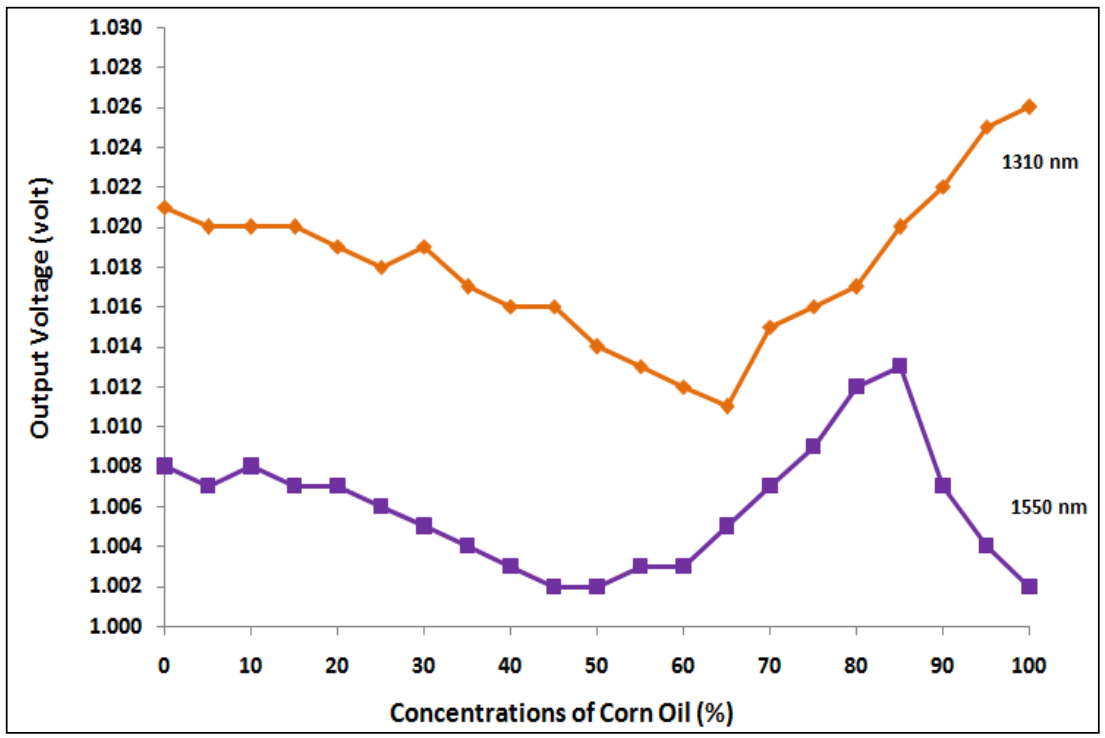

Fig. 2. Fiber-optic sensor responses to different value of corn oil concentrations

The lines graph analysis is recorded in Table 1. These analysis are been separated into several information which are linear range, correlation $\mathrm{R}$, coefficient of determination (COD) and sensitivity. Linear range is a concentration range used from the graph for analysis. For an example, $1310 \mathrm{~nm}$ of light source is separated into two slopes which are $0 \%$ to $65 \%$ of concentration in the first slope and $65 \%$ to $100 \%$ of concentration for the second slope. Correlation $\mathrm{R}$ is represented relation between concentrations value with output voltage. COD is show the percentage of corn oil that affected the voltage result. Sensitivity is show sensitive level capture by fiber optic sensor toward different value of concentrations.

The first analysis is based on slopes for light source $1310 \mathrm{~nm}$ used. Table 1 showed comparisons between two sample slopes with different range of concentrations. It is show that second slope received higher correlation, COD and sensitivity value. From here it is show that fiber optic would be in higher performance. Different concentrations would definitely give an impact to the output voltage. This experiment continues with $1550 \mathrm{~nm}$ of light source and received three slopes analysis. However, the third slope is showed the higher performance of fiber optic as liquid sensor. The third is choose to represent the best result from light source $1550 \mathrm{~nm}$ is because it received higher sensitivity value of the bunch. There is one similarity between these results which is fiber optic sensor could definitely in high performance for high concentration value. However, from both of final results, the third slope of $1550 \mathrm{~nm}$ wave length is choose to be the best result from others. The reason because this slope received correlation $\mathrm{R}$ almost perfect (equal to 1), 94\% of corn oil particles interacted with light source traveled through it and sensitivity value is higher among others. In other words, fiber optic sensor is in high performance for corn oil concentrations detection by using $1500 \mathrm{~nm}$ as light source and operated for concentrations above $85 \%$. 
Table 1. Summary of the performance for the fiber-optic sensor.

\begin{tabular}{|c|c|c|c|c|c|}
\hline \multirow{2}{*}{ Light Sources } & \multicolumn{2}{|c|}{$1310 \mathrm{~nm}$} & \multicolumn{3}{c|}{$1550 \mathrm{~nm}$} \\
\cline { 2 - 6 } & 1st Slope & 2nd Slope & 1st Slope & 2nd Slope & 3rd Slope \\
\hline Linear range (\%) & $0 \sim 65$ & $65 \sim 100$ & $0 \sim 50$ & $50 \sim 85$ & $85 \sim 100$ \\
\hline Correlation R & 0.97 & 0.99 & 0.96 & 0.98 & 0.97 \\
\hline COD (\%) & 94.2 & 98.1 & 92.2 & 95.6 & 94.0 \\
\hline $\begin{array}{c}\text { Sensitivity (volt/ } \\
\%)\end{array}$ & 0.00015 & 0.00042 & 0.00013 & 0.00034 & 0.00072 \\
\hline
\end{tabular}

\section{Conclusion}

Fiber-optic sensor experimentally demonstrated using intensity modulation technique in different of liquid concentrations. The results show the different value of voltage output for each of different concentrations and light sources. The result could be observed in the graft which contains two different slopes (for each of light sources) in the voltage response. In this experiment, the liquid concentrations sensing in the range of 20 different oil concentrations using $1310 \mathrm{~nm}$ and $1550 \mathrm{~nm}$ wavelength for the main input light sources. The sensitivity correlation R and COD are totally depends on these concentration, condition of fiber-optic and light sources. With the $1550 \mathrm{~nm}$ light source, the maximum sensitivity of 0.00072 and COD (linearity) of $94.0 \%$ had been obtained in third slope of fiber-optic sensor, respectively.

Our appreciations and gratitude to Universiti Teknikal Malaysia Melaka (UTeM) for providing the necessity in completing this project. This work was financially supported by UTeM short term grants PJP/2015/FTK(25B)/S01446. To our group members for their great teamwork and our family and friends whom without their support this project would not be a success.

\section{References}

1. S. Binu, V.P. Mahadevan Pillai, N. Chandrarasekaran, Opt. Laser Technol., 39(8), 1537 (2007)

2. G.Y. Chen, M. Ding, T. Newson, G. Brambilla, The Open Optics Journal, 7(1), (2013)

3. Jingyi Lou, Yipei Wang, Limin Tong, Sensors, 14(4), 5823 (2014)

4. Y.S. Chiam, K.S. Lim, S.W. Harun, S.N. Gan, S.W. Phang, Sensor Actuat. A-Phys., 205, 58 (2014)

5. G.Y. Chen, T.P. Newson, G. Brambilla, Opt. Fiber Technol., 19(6), 802 (2013)

6. Y. Zhao, Z.Q. Deng, Q. Wang, Sensor Actuat. B-Chem., 192, 229 (2014) 